\title{
Can masked priming effects be obtained with handwritten words?
}

\author{
Cristina Gil-López • Manuel Perea • \\ Carmen Moret-Tatay • Manuel Carreiras
}

Published online: 13 July 2011

(C) Psychonomic Society, Inc. 2011

One important issue often neglected in the literature on visualword recognition, despite its obvious ecological validity, is how the cognitive system processes handwritten words. Although handscript was the initial and only way of writing/reading until the 15 th century, the vast majority of psycholinguistic experiments use spotless printed words in which, unlike in handwriting, letters are physically separated within each word and the instances of each letter are identical (e.g., compare denied vs. dewied). Not surprisingly, there is some cost associated with the processing of handwritten words (Barnhart \& Goldinger, 2010; Manso de Zuñiga, Evett, \& Humphreys, 1991). Given the inherent noise in the bottom-up information from handwritten words, the cognitive system has to rely more on more effortful, top-down processes (Manso de Zuñiga et al., 1991). Consistent with this interpretation, lexical effects are magnified with handwritten wordsincluding the effects of word frequency, regularity, bidirectional consistency, and imageability (see Barnhart \& Goldinger, 2010).

\footnotetext{
C. Gil-López $(\bowtie) \cdot M$. Carreiras

Basque Center on Cognition, Brain, and Language, Paseo Mikeletegi, 53,

20009, Donostia-San Sebastián, Spain

e-mail: c.gil@bcbl.eu

M. Perea $\cdot$ C. Moret-Tatay

Universitat de València,

Valencia, Spain

C. Moret-Tatay

Universidad Católica de Valencia,

Valencia, Spain

M. Carreiras

IKERBASQUE, Basque Foundation for Science,

Bilbao, Spain
}

The aim of the present study is to investigate how the word-processing system processes handwritten words at the very early moments. To explore the earliest stages of visualword recognition, a highly useful technique is the masked priming paradigm (Forster \& Davis, 1984; see also Grainger, 2008, for a recent review), in which a briefly presented prime word precedes the presentation of a target word. One highly robust and replicated phenomenon in this context is the masked repetition priming effect: Identification times to a target word are consistently faster when it has been briefly preceded by the same word than when it has been preceded by an unrelated word (Forster \& Davis, 1984; see Dehaene et al., 2001, for fMRI evidence; see Carreiras, Duñabeitia, \& Molinaro, 2009, for ERP evidence).

Can handuritten words produce masked priming effects? Clearly, if masked priming occurs with handwritten primes, it will be possible to examine the impact of handwritten words on orthographic, phonological, or morphological processes at the earliest stages of processing using behavioral and/or neurophysiological techniques. Therefore, we believe that it is critical to demonstrate, in the first place, the existence of masked repetition priming with handwritten primes (i.e., the most robust form of masked priming). Previous research has found that masked priming effects are quite abstract in nature; indeed, they can be obtained between upper- and lowercase typed words that are visually dissimilar (e.g., edge-EDGE; see Bowers, Vigliocco, \& Haan, 1998). This would suggest that masked priming should extend between written and typed primes and targets - at least for easily readable prime words (e.g., cable-CABLE). However, the only published experiment on the issue, conducted by Qiao et al. (2010), failed to find a masked repetition priming effect with handwritten primes using a semantic categorization task (i.e., words referring to man-made objects vs. words referring to natural 
objects). More specifically, Qiao et al. found a sizeable masked repetition priming effect at a 50-ms SOA when the prime was presented in a printed font $(31 \mathrm{~ms}$; e.g., alliance-ALLIANCE). In contrast, they failed to find a masked repetition priming effect not only when the prime was written in a difficult handwritten style (e.g., eleimaALLIANCE; a nonsignificant, -8 -ms priming effect), but also when the prime was written in an easy handwritten style (e.g., alliance-ALLIANCE; a -1-ms priming effect). The key issue at stake here is not the outcome with the "difficult" handwritten words (i.e., these words may be difficult to process) but rather the finding with the "easy" handwritten words - these words seem to be easy to process. Indeed, a pilot study conducted by Qiao et al. only revealed an advantage of $31 \mathrm{~ms}$ in naming times of printed words over "easy" handwritten words (533 vs. 564 ms, respectively; see Qiao et al., 2010, Fig. 1).

Given the importance of examining the processing of handwritten words at the early stages of visual-word recognition, we believe that it is imperative to reexamine whether it is possible to obtain masked repetition priming with handwritten primes - in particular, for easily readable handwritten words. Here we did so in a semantic categorization experiment and in a lexical decision experiment. To that end, we recruited a sample of 10 volunteers and asked them to write down a series of 10 words. We then asked four additional judges to choose the "easiest" writing style among the "writers." The individual whose handwriting was easiest to read wrote down the entire set of word/ nonword prime stimuli in the present experiments-these stimuli were scanned and appropriately scaled to match the printed stimuli. A pilot, unprimed lexical decision experiment conducted on 8 undergraduate students - using the materials from Experiment 2 in the present study - revealed that printed lowercase words (e.g., anterior) were responded to faster and more accurately than the handwritten words (e.g., antenior): 591 versus $625 \mathrm{~ms}$ and $2.8 \%$ versus $5.2 \%$ errors, respectively. (The differences were significant in both $F_{1}$ and $F_{2}$ analyses.) Thus, the handwritten stimuli employed in the present experiments were comparable to the "easy handwriting style" (e.g., alliance) employed by Qiao et al. (2010).

In the present study, we followed the standard practice in the literature on masked priming: We selected a large set of nonrepeated target words in the experimental session (320 words in Exp. 1 and 304 words in Exp. 2)-Qiao et al. (2010) used a set of 48 target words that was repeated six times in the experiment. In Experiment 1, we examined whether masked repetition priming for handwritten prime words can be obtained in a semantic categorization task ("does the word refer to a natural object [e.g., mouse] or to a man-made object [e.g., skirt]?"). Although most semantic categorization tends to use more well-defined semantic categories (e.g., "is it an animal?"; see Forster, 2004, for a review), we chose the above-cited semantic categories because these categories were the ones employed by Qiao et al. in their masked repetition priming experiment with handwritten primes. To assess whether masked repetition priming with handwritten words generalizes over tasks, in Experiment 2, we employed a lexical decision task ("is the stimulus a real word?"), which is the more common task in the literature on masked repetition priming.

\section{Experiment 1: Semantic categorization task}

Method

Participants A group of 20 students from the Catholic University of Valencia participated voluntarily in the experiment. All of them had either normal or corrected-tonormal vision and were native speakers of Spanish.

Materials We selected a set of 160 target words that referred to man-made objects (e.g., piano, cable, etc.) and 160 target words that referred to natural objects (e.g., melon, elephant, etc.) from the Spanish database (Davis \& Perea, 2005). The mean lengths for the words referring to man-made objects and natural objects were 6.25 and 6.25 letters (range: 5-9), the mean word frequencies per million were 15.4 and 15.4, and the mean numbers of substitutionletter neighbors (i.e., Coltheart's $N$ ) were 1.4 and 1.5, respectively. The list of stimuli is available at www.uv.es/ mperea/hand_rep.pdf. The targets were presented in uppercase and were preceded by word primes in lowercase that were (1)the same as the target (identity condition) for instance, melón-MELÓN (melón is the Spanish for melon) or torre-TORRE (torre is the Spanish for tower) or (2) completely unrelated to the target (unrelated condition), belonging to a different category and with the same length-for instance, cable-MELÓN (cable is the Spanish for cable) or limón-TORRE (limón is the Spanish for lemon). (We chose unrelated primes belonging to semantic categories different from that of the target to parallel the prime/target pairs employed by Qiao et al., 2010.) The primes in the identity and unrelated conditions were presented either in a standard printed font (11-point Century; e.g., melón-MELÓN, cable-MELÓN, torreTORRE, limón-TORRE) or in natural handwritten form (e.g., melón-MELÓN, cable-MELÓN, torre-TORRE, limón TORRE). The handwritten words were resized by applying a scaling factor so that their size was comparable to that of the standard printed font. Target stimuli were presented in uppercase 18-point Courier New font. Four lists of stimuli were created to counterbalance the materials across the two factors in the experiment; this way, each target 
appeared only once in each list, but in different priming conditions for different lists. Five of the participants were randomly assigned to each list.

Procedure Participants were tested individually in a quiet room. Presentation of the stimuli and recording of reaction times (RTs) were controlled by a Windows-based computer using DMDX (Forster \& Forster, 2003). On each trial, a forward mask consisting of a string of hash marks (\#s) was presented for $500 \mathrm{~ms}$ in the centre of the screen monitor. Then, the lowercase prime was presented for $50 \mathrm{~ms}$ and was followed immediately by the presentation of the target stimulus in uppercase. RTs were measured from target onset to the participant's response. The strings were presented centered in black on a white background. Participants were instructed to press one button if the word referred to a manmade object and another if the word referred to a natural object. They were not informed of the presence of the lowercase items. Each participant received a different order of trials. When asked after the experiment, participants did not report having seen any prime stimuli. The whole experimental session lasted for about $25 \mathrm{~min}$.

\section{Results and discussion}

Incorrect responses $(7.0 \%$ of the data) and RTs less than $250 \mathrm{~ms}$ or greater than $2,000 \mathrm{~ms}(1.9 \%$ and $1.4 \%$ of the data for the man-made and the natural objects, respectively) were excluded from the RT analysis. The mean RTs and error percentages from the participant analysis are presented in Table 1. ANOVAs based on the participant and item mean correct response latencies were conducted based on a 2 (Target Type: man-made object, natural object) x 2 (PrimeTarget Relatedness: identity, unrelated) x 2 (Prime Type: printed, handwritten) $x 4$ (List: 1-4) design. In this and in the

Table 1 Mean semantic categorization times (in milliseconds) and percentage of errors (in parentheses) for word targets in Experiment 1

\begin{tabular}{lll}
\hline & \multicolumn{2}{l}{ Type of Prime } \\
\cline { 2 - 3 } & Printed & Handwritten \\
\hline Man-Made Objects & & \\
Related & $897(5.8)$ & $891(4.3)$ \\
Unrelated & $929(4.5)$ & $921(5.6)$ \\
Priming (U - R) & $32(-1.3)$ & $30(1.4)$ \\
Natural Objects & & \\
Related & & $884(8.5)$ \\
Unrelated & $867(8.6)$ & $881(9.0)$ \\
Priming (U - R) & $17(0.9)$ & $-3(0.5)$ \\
\hline
\end{tabular}

subsequent experiment, List was included as a dummy factor to extract the variance due to the error associated with the counterbalancing lists (Pollatsek \& Well, 1995).

The ANOVA on the latency data showed that, on average, responses to words referring to man-made objects were $31 \mathrm{~ms}$ slower than responses to words referring to natural objects, $F_{1}(1,16)=7.91, M S E=4,734.6, p<.02 ; F_{2}(1,312)=9.27$, $M S E=30,199.6, p<.004$. More importantly, the word targets preceded by an identity prime were responded to $19 \mathrm{~ms}$ faster than the word targets preceded by an unrelated prime, $F_{1}(1,16)=10.00, M S E=1,391.3, p<.007$; $F_{2}(1,312)=13.88, M S E=9,868.6, p<.001$. The interaction between target type and relatedness was significant, $F_{1}(1,16)=8.83, M S E=673.8, p<.01 ; F_{2}(1,312)=4.55$, $M S E=9,868.6, p<.04$. This interaction reflected that the effect of relatedness was robust when the words referred to man-made objects $\left(34 \mathrm{~ms} ; F_{1}(1,16)=14.16, M S E=\right.$ $1,344.4, p<.003 ; F_{2}(1,156)=18.43, M S E=9,194.3$, $p<.001)$, whereas the repetition priming effect was not significant when the words referred to natural objects (a nonsignificant 6 -ms effect; $F_{1}(1,16)=1.16, M S E=$ $\left.720.8, p>.25 ; F_{2}(1,156)=1.19, M S E=9,914.0, p>.20\right)$. Note that although there seemed to be some repetition priming for the printed primes relative to the handwritten primes (17 vs. $-3 \mathrm{~ms}$ ), the interaction between relatedness and prime type for words referring to natural objects did not approach significance $\left(F_{1}<1 ; F_{2}(1,156)=2.61, M S E=\right.$ $10,507.0, p>.10)$. The other interactions did not approach significance (all $p \mathrm{~s}>.14$ ).

The ANOVA on the error data showed that, on average, words referring to man-made objects produced fewer errors than the responses to words referring to natural objects $\left(5.0 \%\right.$ vs. $8.9 \%$, respectively), $F_{1}(1,16)=21.42, M S E=$ $28.0, p<.001 ; F_{2}(1,312)=17.93, M S E=267.9, p<.001$. None of the other effects/interactions approached significance (all $p s>.25$ ).

The present semantic categorization experiment revealed that, for words referring to man-made objects (e.g., book, pencil, wall), there is a large masked repetition priming effect for both printed and handwritten primes (32 vs. $30 \mathrm{~ms}$, respectively; printed primes: $F_{1}(1,16)=5.61, M S E=$ $1,824.6, p<.04 ; F_{2}(1,156)=6.53, M S E=10,752.6$, $p<.015$; handwritten primes: $F_{1}(1,16)=5.38, M S E=$ $1,638.0, p<.04 ; F_{2}(1,156)=11.17, M S E=9,003.7$, $p<.002)$. Thus, handwritten primes can produce a sizeable masked priming effect in the latency data.

One somewhat unexpected finding, however, was the absence of a sizeable masked repetition priming effect for words referring to natural objects: The overall priming effect was a nonsignificant 6-ms effect. What we should note here is that Qiao et al. (2010) did not include Target Type (man-made vs. natural object) as a factor in the statistical analyses (or in the tables with the descriptive 
indexes), and no information was provided in their article as to whether there were differential priming effects for words referring to man-made and natural objects. Perhaps the failure to obtain an overall priming effect for handwritten words in the Qiao et al. study was related to the fact that the two categories do not behave in exactly the same way. Note here that if we average the priming effects for handwritten primes, the size of the repetition priming effect was quite small: $7 \mathrm{~ms}$, which is somewhat comparable to the -1 -ms priming effect reported by Qiao et al. with easyto-read handwritten primes.

The question now is whether masked repetition priming effects with handwritten primes can be obtained in the most popular word laboratory identification task: the lexical decision task. This is the goal of Experiment 2. Furthermore, in Experiment 2 we also manipulated (withinsubjects) the stimulus onset asynchrony (SOA) (33 and $50 \mathrm{~ms}$ ) to examine the time course of the activation of handwritten words.

\section{Experiment 2: Lexical decision task}

\section{Method}

Participants A group of 16 students from the University of Valencia participated voluntarily in the experiment. All of them had either normal or corrected-to-normal vision and were native speakers of Spanish.

Materials We selected a set of 304 target words from the Spanish database (Davis \& Perea, 2005). The mean length was 6.5 letters (range 5-8), the mean word frequency per million was 37.8 , and the mean number of substitution-letter neighbors (i.e., Coltheart's $N$ ) was 0.86 . A list of 304 unrelated prime words matched in word frequency and length to the target words - on a pairwise basis - was also generated. The list of stimuli is available at www.uv.es/mperea/hand_rep.pdf. The targets were presented in uppercase and were preceded by primes in lowercase that were (1) the same as the target (identity condition)-for instance, otoño-OTOÑO (otoño is the Spanish for autumn) - or (2) completely unrelated to the target (unrelated condition)-for instance, arena-OTOÑO. For the purposes of the lexical decision task, 304 nonword targets were created (mean length 6.5 letters, range 5-8), as well as 304 additional nonwords to act as unrelated primes. These nonwords had been created by changing two letters from Spanish words that did not form part of the word list in the experiment. The mean number of substitution-letter neighbors of the nonword targets (Coltheart's $N=0.38$ ) was similar to that for the words. Nonword targets were preceded by identity nonword primes or by unrelated nonword primes (e.g., arralARRAL vs. mesco-ARRAL; navadosa-NAVADOSA vs. gazpanto- NAVADOSA). The primes in the identity and unrelated conditions were presented in a standard printed font (11-point Century; e.g., otoño-OTOÑO, arena$O T O \tilde{N} O)$ or in natural handwritten form (e.g., otoñoOTOÑO, arena-OTOÑO). The handwritten prime words were easily legible and were written by the same individual as in Experiment 1. As in Experiment 1, the handwritten stimuli were resized by applying a scaling factor so that their size was comparable to that of the standard printed font. Target stimuli were presented in 18point Courier New font. Eight lists of stimuli were created to counterbalance the materials across the three factors in the experiment, so that each target appeared only once in each list, but in different priming conditions for different lists. Different participants were assigned to each list.

Procedure This was the same as in Experiment 1, except that the SOA was set at either 33 or $50 \mathrm{~ms}$ (depending of the SOA level) and participants were instructed to press a button labeled "si" [yes] if the string formed an existing Spanish word and a button labeled "no" if the string was a nonword.

\section{Results and discussion}

Incorrect responses $(3.9 \%$ and $4.4 \%$ of the data for word and nonword targets, respectively) and RTs less than $250 \mathrm{~ms}$ or greater than $1,500 \mathrm{~ms}(1.1 \%$ of the data for word targets and $4.8 \%$ of the data for nonword targets) were excluded from the latency analysis. ${ }^{1}$ The mean RTs and error percentages from the participant analysis are presented in Table 2. ANOVAs based on the participant and item mean correct response latencies were conducted based on a 2 (Prime-Target Relatedness: identity, unrelated) x $2(\mathrm{SOA}: 33 \mathrm{~ms}, 50 \mathrm{~ms}) \times 2$ (Prime Type: printed, handwritten) x 8 (List: 1-8) design. The factors Prime-Target Relatedness, SOA, and Prime Type were manipulated within subjects and within items. Error rates were very low (3.9\% for words) and were not analyzed further.

Word data The ANOVA on the latency data showed that word targets preceded by an identity prime were responded

\footnotetext{
${ }^{1}$ We employed a 1,500-ms cutoff for the lexical decision task because RTs were substantially faster than in the semantic categorization task, and the percentage of trimmed values for word targets was comparable to that in Experiment 1. With respect to the nonword targets, a 2,000ms cutoff produced essentially the same pattern of data as that reported here.
} 
Table 2 Mean lexical decision times (in milliseconds) and percentage of errors (in parentheses) for word and pseudoword targets in Experiment 2

\begin{tabular}{|c|c|c|}
\hline & \multicolumn{2}{|c|}{ Type of Prime } \\
\hline & Printed & Handwritten \\
\hline \multicolumn{3}{|l|}{ Word Targets } \\
\hline \multicolumn{3}{|l|}{ 33-ms SOA } \\
\hline Related & $651(3.9)$ & $655(3.1)$ \\
\hline Unrelated & $674(4.1)$ & $673(6.4)$ \\
\hline Priming $(U-R)$ & $23(0.2)$ & $18(3.3)$ \\
\hline \multicolumn{3}{|l|}{ 50-ms SOA } \\
\hline Related & $629(2.6)$ & $652(3.1)$ \\
\hline Unrelated & $674(4.4)$ & $683(3.8)$ \\
\hline Priming $(U-R)$ & $45(1.8)$ & $31(0.7)$ \\
\hline \multicolumn{3}{|l|}{ Nonword Targets } \\
\hline \multicolumn{3}{|l|}{ 33-ms SOA } \\
\hline Related & $776(6.4)$ & 793 (3.8) \\
\hline Unrelated & $785(5.4)$ & $781(2.6)$ \\
\hline Priming $(U-R)$ & $9(-1.0)$ & $-12(-1.2)$ \\
\hline \multicolumn{3}{|l|}{ 50-ms SOA } \\
\hline Related & $791(3.5)$ & $788(5.6)$ \\
\hline Unrelated & $792(3.8)$ & 783 (3.9) \\
\hline Priming (U - R) & $1(0.3)$ & $-5(-1.6)$ \\
\hline
\end{tabular}

to $29 \mathrm{~ms}$ faster than the word targets preceded by an unrelated prime, $F_{1}(1,8)=39.91, M S E=1,351.1, p<.001$; $F_{2}(1,282)=54.78, M S E=11,754.1, p<.001 .^{2}$ The interaction between SOA and relatedness was also significant, $F_{1}(1,8)=17.26, M S E=281.7, p<.004 ; F_{2}(2,282)=4.46$, $M S E=9,993.4, p<.04$, which reflected that the effect of relatedness was greater at the 50 -ms SOA than at the $33-\mathrm{ms}$ SOA (38 vs. $20 \mathrm{~ms}$, respectively). Importantly, the effects of masked repetition priming were sizeable, and similar in magnitude, for the printed primes (34 ms), $F_{1}(1,8)=27.70$, $M S E=1,308.9, p<.001 ; F_{2}(1,286)=17.12, M S E=$ $10,533.0, p<.001$, and for the handwritten primes (24 ms), $F_{1}(1,292)=39.22, M S E=11,497.3, p<.001$, as deduced from the lack of interaction between relatedness and prime type, $F_{1}(1,8)=1.10, M S E=1,245.3, F_{2}<1$. The other effects/interactions were not significant (all $p$ s $>.18$ ).

Nonword targets The ANOVA on the latency data only showed an interaction between prime type and relatedness in the analysis by participants, $F_{1}(1,8)=9.17, M S E=$ $316.1, p<.02 ; F_{2}(1,273)=3.39, M S E=17,986.8, p=$

\footnotetext{
${ }^{2}$ Because of the large number of counterbalancing lists, there were a few missing cells in the RT analysis over items in the word data (14 out of 304 items).
}

.067. This reflected nonsignificant 4-ms priming for printed primes (both $F \mathrm{~s}<1$ ) and a nonsignificant -8.5 -ms priming effect for handwritten primes (both $p \mathrm{~s}>.25$ ). The other effects were not significant (all $p \mathrm{~s}>.20$ ).

The present experiment revealed a large masked identity priming effect for both printed prime words and handwritten prime words ( 34 vs. $24 \mathrm{~ms}$, respectively). It is important to note here that the presence of a robust masked repetition priming effect with handwritten primes in the lexical decision task is not an empirical anomaly: A replication of the experiment at a $50-\mathrm{ms}$ SOA $(N=18)$ revealed a significant repetition priming effect for both printed primes (44 ms: 605 vs. $649 \mathrm{~ms}$ for the identity and unrelated conditions, respectively; both $p \mathrm{~s}<.001$ ) and handwritten primes $(30 \mathrm{~ms} ; 621$ vs. $651 \mathrm{~ms}$ for the identity and unrelated conditions; both $p \mathrm{~s}<.001)^{3}$

\section{General discussion}

The present masked priming experiments were designed to shed some light on the intricacies of handwritten words at the earliest stages of word processing. The findings were clear. First, in the semantic categorization task, we found a sizeable masked repetition priming effect with handwritten prime words for man-made words $(31 \mathrm{~ms}$; e.g., melón$M E L O ́ N$ faster than cable-MELÓN) - a priming effect very similar to that obtained with printed words $(32 \mathrm{~ms}$; melón$M E L O ́ N$ faster than cable-MELÓN). Second, unlike in the words referring to man-made objects, the masked repetition priming effect in semantic categorization for words referring to natural objects was small and nonsignificant - for both printed words and handwritten words. Third, in the lexical decision task, we obtained a robust (around 24-ms) masked repetition priming effect with handwritten prime

\footnotetext{
${ }^{3}$ In addition, we examined whether word length could modulate the magnitude of masked repetition priming with handwritten fonts. The magnitudes of masked priming effects were very similar for the shorter and the longer words - both in the present experiment and in the replication. A similar pattern occurred when we carried out some post hoc analyses on the potential effect of word frequency on the magnitude of masked priming effects. Finally, we should also note here that, in a recent study comparing the yes/no and the go/no-go procedures in lexical decision, Perea, Gomez, and Fraga, (2010) reported a significant $11-\mathrm{ms}$ repetition priming effect for nonword targets. In the present experiment, we found a 9-ms and a 1-ms effect for printed primes at the 33- and 50-ms SOAs, respectively, which were not significant. In the replication experiment, we found a (nonsignificant) 9-ms priming effect for nonword targets. We believe that the empirical discrepancies regarding the estimation of the magnitudes of masked priming effects with nonword targets are related to the fact that responses to nonwords in lexical decision may be made on the basis of different sources of information-some of which may produce priming, and others may not.
} 
words (e.g., otoño-OTOÑO faster than arena-OTOÑO), which was only slightly less than that obtained with printed words $(34 \mathrm{~ms}$; e.g., otoño-OTOÑO faster than arenaOTOÑO). When we take these results together, we have demonstrated that handwritten primes - at least when they are easily readable (e.g., anterior, arena, cabe) - can produce a sizeable masked repetition priming effect in the latency data of semantic categorization and lexical decision and tasks. ${ }^{4}$

The present study has one obvious implication: A powerful tool such as the masked priming technique can be employed in experiments with handwritten primes. Thus, it is possible to examine the potential impact of handwritten words on cognitive processes at the earliest stages of processing with this technique (see Grainger, 2008, for a review); easy-to-read handwritten words can produce reliable masked priming effects, as demonstrated here. Thus, the present findings support the view that masked priming effects have an abstract nature (see Bowers et al., 1998, for evidence with visually dissimilar primes). We acknowledge, however, that hard-to-read handwritten words such as ellime may not produce a large level of activation at the word level at the early stages of processing - in a way similar to highly degraded speech signal during auditory word recognition (see Barnhart \& Goldinger, 2010, for a discussion of the relationships between handscript and speech).

Before concluding this article, it may be important to briefly discuss one unexpected finding that occurred in the semantic categorization experiment. Previous research with the semantic categorization task has focused on welldefined categories such as "does the word refer to an animal?", and the typical finding is the presence of a large masked repetition priming effect for both exemplars and nonexemplars-although there is some variability concerning the magnitude of the priming effects (see, e.g., Forster, 2004). In Experiment 1, we employed the same categories as Qiao et al. (2010): words referring to manmade versus natural objects. Regardless of the type of prime (which did not interact with the effects of interest), masked repetition priming effects in this experiment were substantially greater for words referring to man-made objects than for words referring to natural objects (31 vs. $7 \mathrm{~ms}$, respectively). (Note that Qiao et al. did not report/ analyze their findings as a function of semantic category.) It is unclear to us why this was the case, although we suspect that it is related to the fact that participants had trouble

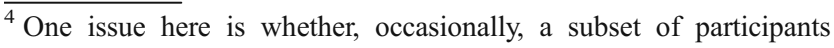
could have had some form of rudimentary awareness of the prime stimuli at the 50-ms SOA, despite the fact that they did not report conscious awareness of the primes after the experiment. In Experiment 2, handwritten primes produced a significant masked repetition priming effect not only at the 50-ms SOA, but also at the 33-ms SOA. We believe that this rules out a "partial awareness" account as an explanation for the present data.
}

making decisions on a broad, heterogeneous category such as "natural objects" and were unsure how to respond to these words - indeed, error rates in that category were twice those for the "man-made" category (see Table 1). One other possibility is that the "man-made" category was the default category (i.e., a "yes" response) for most participants, so that responses for the "natural objects" could have been due to a temporal deadline for "no" responses. Note that a similar argument has been made to explain why masked repetition priming is small and (usually) unreliable for nonword targets in a lexical decision task (see Forster, 1998) - if this mechanism is correct, a go/no-go task for "natural objects" should provide a sizeable masked repetition priming effect (see Siakaluk, Buchanan, \& Westbury, 2003). Although a thorough examination of this issue would go beyond the scope of the present study, a sensible strategy for future semantic categorization experiments would be to focus on a well-defined category (e.g., "is it an animal or not?") rather than on a binary choice task with two nonexhaustive, broad, and heterogeneous categories ("does the word refer to a man-made object or a natural object?").

In sum, handwriting word recognition raises a number of critical issues about how the brain is able to deal with a rather intricate input. The present study has shown that easyto-read handuriten words can activate the appropriate lexical entry when using the masked priming technique across two different experimental tasks: semantic categorization and lexical decision. In this way, researchers can examine in detail the activation produced by handwritten words in the early stages of processing (e.g., at the orthographic, phonological, or morphological level) across a variety of behavioral and and/or neurophysiological techniques.

Author Note This research has been partially supported by Grants PSI2009-08889/PSIC, PSI2008-04069/PSIC, and CONSOLIDERINGENIO2010 CSD2008-00048 from the Spanish Government. We thank Jeff Bowers, Ken Forster, Glenn Bodner, and one anonymous reviewer for their comments and suggestions on a previous version of the manuscript.

\section{References}

Barnhart, A. S., \& Goldinger, S. D. (2010). Interpreting chickenscratch: Lexical access for handwritten words. Journal of Experimental Psychology. Human Perception and Performance, 36, 906-923. doi:10.1037/a0019258

Bowers, J. S., Vigliocco, G., \& Haan, R. (1998). Orthographic, phonological, and articulatory contributions to masked letter and word priming. Journal of Experimental Psychology. Human Perception and Performance, 24, 1705-1719. doi:10.1037/ 0096-1523.24.6.1705

Carreiras, M., Duñabeitia, J. A., \& Molinaro, N. (2009). Consonants and vowels contribute differently to visual word recognition: ERPs of relative position priming. Cerebral Cortex, 19, 2659 2670. doi:10.1093/cercor/bhp019 
Davis, C. J., \& Perea, M. (2005). BuscaPalabras: A program for deriving orthographic and phonological neighborhood statistics and other psycholinguistic indices in Spanish. Behavior Research Methods, 37, 665-671. doi:10.3758/BF03192738

Dehaene, S., Naccache, L., Cohen, L., Le Bihan, D., Mangin, J.-F., Poline, J.-B., et al. (2001). Cerebral mechanisms of word masking and unconscious repetition priming. Nature Neuroscience, 4, 752-758. doi:10.1038/89551

Forster, K. I. (1998). The pros and cons of masked priming. Journal of Psycholinguistic Research, 27, 203-233. doi:10.1023/ A:1023202116609

Forster, K. I. (2004). Category size effects revisited: Frequency and masked priming effects in semantic categorization. Brain and Language, 90, 276-286. doi:10.1016/S0093-934X(03)00440-1

Forster, K. I., \& Davis, C. (1984). Repetition priming and frequency attenuation in lexical access. Journal of Experimental Psychology. Learning, Memory, and Cognition, 10, 680-698. doi:10.1037/ 0278-7393.10.4.680

Forster, K. I., \& Forster, J. C. (2003). DMDX: AWindows display program with millisecond accuracy. Behavior Research Methods, Instruments, and Computers, 35, 116-124. doi:10.3758/BF03195503

Grainger, J. (2008). Cracking the orthographic code: An introduction. Language and Cognitive Processes, 23, 1-35. doi:10.1080/ 01690960701578013
Manso de Zuñiga, C., Evett, L. J., \& Humphreys, G. W. (1991). Reading by type and by hand: The effects of typography on visual word recognition. In D. Besner \& G. W. Humphreys (Eds.), Current issues in word recognition (pp. 10-33). Hillsdale, NJ: Erlbaum.

Perea, M., Gómez, P., \& Fraga, I. (2010). Masked nonword repetition effects in yes/no and go/no-go lexical decision: A test of the evidence accumulation and deadline accounts. Psychonomic Bulletin and Review, 17, 369-374. doi:10.3758/ PBR.17.3.369

Pollatsek, A., \& Well, A. D. (1995). On the use of counterbalanced designs in cognitive research: A suggestion for a better and more powerful analysis. Journal of Experimental Psychology. Learning, Memory, and Cognition, 21, 785-794. doi:10.1037/02787393.21.3.785

Qiao, E., Vinckier, F., Szwed, M., Naccache, L., Valabrègue, R., Dehaene, S., et al. (2010). Unconsciously deciphering handwriting: Subliminal invariance for handwritten words in the visual word form area. NeuroImage, 49, 1786-1799. doi:10.1016/j. neuroimage.2009.09.034

Siakaluk, P. D., Buchanan, L., \& Westbury, C. (2003). The effect of semantic distance in yes/no and go/no-go semantic categorization tasks. Memory \& Cognition, 31, 100-113. doi:10.3758/ BF03196086 